\title{
ISOTIAZOLINAS: IMPORTANCIA EN EL DESARROLLO DE DERMATITIS DE CONTACTO PROFESIONAL
}

\author{
ENRIQUE CABRERA FERNÁNDEZ*, GREGORIO MORENO MANZANO*, LUCÍA BARCHINO ORTIZ**, \\ VERÓNICA PEREZ GARCÍA***, FELIPE HERAS MENDAZA****, LUÍS CONDE SALAZAR****. \\ * Médicos en formación MIR en Medicina del Trabajo. Asepeyo, Mutua de Accidentes de Trabajo \\ y Enfermedades Profesionales de la Seguridad Social n ${ }^{\circ} 151$. \\ ** Médico en formación MIR en Dermatología. Hospital Universitario San Carlos de Madrid. \\ *** Médico en formación MIR en Cirugía Maxilofacial. Hospital Universitario Gregorio Marañón. \\ **** Unidad de Dermatología Laboral. Instituto de Salud Carlos III. Pabellón 8 \\ Universidad Complutense. Madrid
}

\section{RESUMEN}

El Kathon es un conservante resultante de la mezcla de dos isotiazolinonas. Se utiliza preferentemente en la industria cosmética a concentraciones 3-15 ppm. También se ha impuesto su presencia en productos de limpieza de uso doméstico y actualmente a concentraciones más altas en preparados de uso industrial como aceites de corte, emulsiones de látex, pinturas al temple, reveladores de fotografía y radiografía, aceites para motores Diesel...Todo ello hace que sea un sensibilizante importante en nuestra vida privada y profesional. OBJETIVOS: 1 . Demostrar la relación del Kathon como sustancia sensibilizante en el desarrollo de dermatitis de contacto. 2. Demostrar la relación entre Kathon y dermatitis de contacto de origen laboral y determinadas profesiones.METODOLOGIA: Estudio descriptivo: revisión de las historias clínicas de los pacientes que fueron vistos por el Servicio de Dermatología Laboral del Instituto Nacional de Medicina y Seguridad en el Trabajo durante los años 2004-2006; 2333 pacientes, remitidos por diferentes lesiones dermatológicas con posible relación laboral. Buscaremos los sensibilizados a Kathon y analizaremos las variables: sexo, edad, profesión, enfermedades dermatológica de base, localización de las lesiones, manejo de productos que contengan Kathon. Realizaremos el análisis estadístico con el programa SPSS. RESULTADOS: De las 110 positividades a Kathon, 32 son relacionadas con el trabajo $(37,2 \%)$. Podemos sumarles otros 13 casos dudosos $(15,11 \%)$ y de estos casos positivos, el $46,6 \%$ fueron varones. CONCLUSIONES: El Kathon es un conservante muy utilizado en el mundo laboral, y son precisamente las profesiones que más productos con Kathon usan, las que mayor número de casos registran.

\section{PALABRAS CLAVES}

Isotiazolinas, dermatitis de contacto, Kathon, profesional, cosmética.

\section{ABSTRACT}

The Kathon is a preservative obtained of the mixture of two isothiazolinones. It is preferently used in the cosmetic industry at 3-15 ppm concentrations. Its presence has increased from the end of the 70's in Europe and from the middle of the 80's in USA. The product presence has also imposed in house-cleaning use and at higher concentrations in the industrial use as motor oils, latex emulsions, painting oils, revealers for photography and x-ray, oils for diesel engines... All of these make it to be an important irritating product in our private and professional life. OBJECTIVES: 1 . To demonstrate the relation of the Kathon like irritating substance in producing contact dermatitis. 2. To demonstrate to the relation between Kathon and contact dermatitis of labor origin and certain professions. METHODOLOGY: Descriptive study: revision of clinical histories of the patients who have been observed in the Department of Labor Dermatology of the National Institute of Medicine and Labor Security during years 2004-2006; 2333 patients, found with different dermatological injuries with possible labor relation. We will look for the Kathon-sensitive persons and we will analyze the variables: sex, age, dermatological profession, dermatological diseases, location of the injuries, product handling that contains Kathon. We will make the statistical analysis with program SPSS. RESULTS: Of the 110 positive reactions to Kathon, 32 are related to the labour activities (29.09\%). We can add other 13 doubful cases to them $(11.81 \%)$ and of these positive cases, $46.6 \%$ were men. CONCLUSIONS: The Kathon is an additive very used in the labour ambit. The professions that are using more products with Kathon are those with greater number of cases registered.

\section{KEY WORDS}

Isotiasolines, contact dermatitis, Kathon, professional, cosmetics. 


\section{INTRODUCCIÓN}

El Kathon CG (Cosmetic Grade) es el nombre comercial de la primera mezcla de isotiazolinas, fue sintetizado por la industria química a mediados de los 60; sus características principales son su escaso precio y su gran eficacia a bajas concentraciones frente a bacterias (Gram positivas y negativas), hongos, levaduras y algas, propiedades difíciles de conseguir con otros productos químicos similares ${ }^{1}$. Su composición es el resultado de la mezcla de un líquido amarillo claro soluble en agua y alcoholes de bajo grado y que consta de los siguientes ingredientes:

1. $1,5 \%$ de ingrediente activo, que son una mezcla de

- 1,125\% de MI (2 metil 4 isotiazolin 3 ona).

- 0,375\% de MCI (5 cloro-2 metil 4 isotiazo-lin3 ona)

2. $23 \%$ de estabilizadores (cloruro y nitrato de magnesio).

\section{3. $75,5 \%$ de agua.}

Datos Físico-Químicos: Destaca por tener una Densidad: $1.190-1.210 \mathrm{~g} / \mathrm{ml}$, ser miscible tanto en agua, como alcoholes de bajo peso molecular y glicoles. Es compatible con surfactantes aniónicos, catiónicos y no-iónicos, y con emulgentes. Los ingredientes activos pueden ser desactivados por soluciones concentradas de aminas ${ }^{2}$.

Sinónimos del Kathon: 2-metal-4-isothiazolin3-one; Acticide; Algucid CH50; Amerstat 250; Euxyl K 100; Cl+Me-isothiazolinone; Fennosan IT 21; Grotan K; Grotan TK2; GR 856 Izolin; Katon 886 MW; Katon CG; Kathon DP; Kathon LX ; Kathon UT; Kathon WT ; Mergal K7; Metat GT ; Metatin GT; Methylisothiazolinone; Mitco CC 32 L; Paretol; Parmetol DF 35, -DF 12, -A23, -K50, K40, -DF 18; P3 Multan D; Piror P109; Special Mx 323

Por su condición de biocida, el Katon CG se utiliza preferentemente en la industria cosmética, para la conservación de productos cosméticos tales como champúes, geles, cremas para la piel y lociones $^{3}$, a concentraciones de $0,02-0,1 \%$, lo que equivale a 3-15 ppm. Su presencia ha ido en aumento desde finales de los 70 en Europa y mediados de los 80 en EE.UU., encontrándose principalmente en preparados tipo rinse-off (productos que serán aclarados), como son los champús, geles de baño, y los preparados tipo stay-on (productos que no serán aclarados, como cremas y leches limpiadoras) ${ }^{4}$. También se ha impuesto su presencia en productos de limpieza de uso doméstico y actualmente a concentraciones más altas en preparados de uso industrial como aceites de corte, emulsiones de látex, pinturas al temple, reveladores de fotografía y radiografía, aceites para motores Diesel... $5,6,7,8,9$

Todo ello hace que sea un sensibilizante importante tanto en nuestra vida privada como en la vida profesional., provocando dermatitis de contacto alérgica, sobretodo a concentraciones superiores a las recomendadas ${ }^{10}$.

Usos del Kathon: Adhesivos y gomas, Baños de espuma, Agentes limpiadores, Cosméticos, Champúes, Detergentes, Suavizantes de ropa, Geles de cuerpo y pelo, Productos de limpieza de casa, Emulsiones de látex, Mascaras, Fluidos de corte en metales, Toallas de limpieza húmedas, Pinturas, Pesticidas, Productos de sanidad, Pigmentos, Abrillantadores, Conservantes, Tintas de imprenta, Radiografía, Jabones. ${ }^{11,12,13,14,15}$

\section{MATERIAL Y METODOS}

Se ha realizado una revisión de las historias clínicas de los pacientes que fueron vistos por el Servicio de Dermatología Laboral del Instituto Nacional de Medicina y Seguridad en el Trabajo (INMST) durante los años 2003-2006. Los pacientes vistos en este periodo fueron un total de 2333 pacientes, remitidos por diferentes lesiones dermatológicas con posible relación laboral.

A todos estos pacientes se les realizó una historia personal y clínico-laboral detallada preguntándoles sobre productos que manejaban en su vida personal y durante su trabajo. En función de la historia obtenida se les parcheaban baterías específicas, baterías con sustancias propias, etc, pero siempre la batería standard que contenía los alergenos del TRUE TEST de CHEMOTECHNIQUE DIAGNOSTICS.

En dicha batería el alérgeno 23 corresponde al Kathon y se encuentra en una concentración de 100 partes por millón.

Los parches fueron puestos en la espalda y fijados mediante tiras adhesivas. Se levantaron a las 48 horas para una primera lectura y a las 96 horas para una segunda y definitiva lectura. Los diferentes resultados fueron anotados en la historia clínica del paciente. 


\section{RESULTADOS}

Del total de pacientes parcheados durante estos dos años ( $\mathrm{n}=2333$ ), el número de sensibilizaciones al Kathon, fue de 110 personas (4,71\%), de estas 53 fueron mujeres y 57 hombres (48,18\% y $51,82 \%$ respectivamente). De los 24 pacientes que dieron solamente positividad frente a Kathon y no a otros sensibilizantes, 14 eran hombres (58.33\%) y el resto mujeres (41.67\%). (Tabla 1)
En cuanto a la relación laboral (Figura 1), que es lo que más nos interesa, podemos ver que de las 110 positividades, 32 son relacionadas con el trabajo (29,09\%). Podemos sumarles otros 13 casos $(11,81 \%)$ en los que hay sustancias sensibilizantes tanto en el trabajo como en la vida privada, luego no podemos asegurar que la sensibilización sea solamente laboral. De estos 45 positivos, 21 corresponden a varones y 24 a mujeres (46,6\% y 53,3\% respectivamente).

Tabla 1. Distribución por sexo de las sensibilizaciones encontradas.

\begin{tabular}{|l|l|l|l|}
\hline & MASCULINO & FEMENINO & TOTAL \\
\hline + Khaton y otros & 43 & 43 & 86 \\
\hline + Solo Khaton & 14 & 10 & 24 \\
\hline
\end{tabular}

Figura 1: Relación laboral de los casos encontrados.
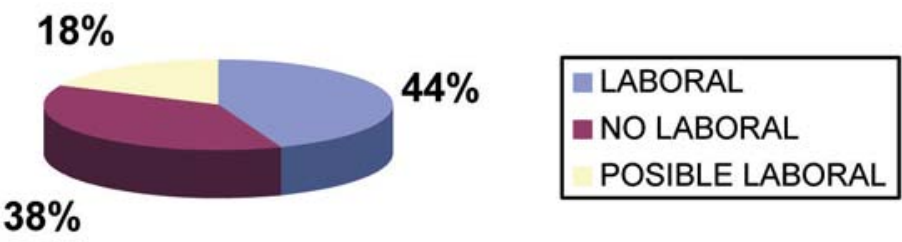

En la distribución de los casos por sexo, vemos profesiones de distribución prácticamente masculinas (mecánicos) y otras femeninas (Peluquería, limpieza) (figura 2)

En cuanto a la edad de los pacientes afectados (figura 3) vemos que en su mayoría están comprendidas entre los 25 años a los 55 años, lo que normalmente corresponde a la mitad de la vida laboral de la población activa. Observándose también que la

aparición de los síntomas es más precoz en las mujeres (a edades más tempranas).

Los pacientes acudieron principalmente con lesiones Eritematosas, descamación y eczema. Estas lesiones corresponden y son propias de las principales patologías de las que finalmente fueron diagnosticados tras las pruebas realizadas (sensibilización al Kathon, y el Eczema alérgico de contacto profesional). (Figuras 4 y 5).

Figura 2: Distribución por sexo y profesión de los casos positivos.

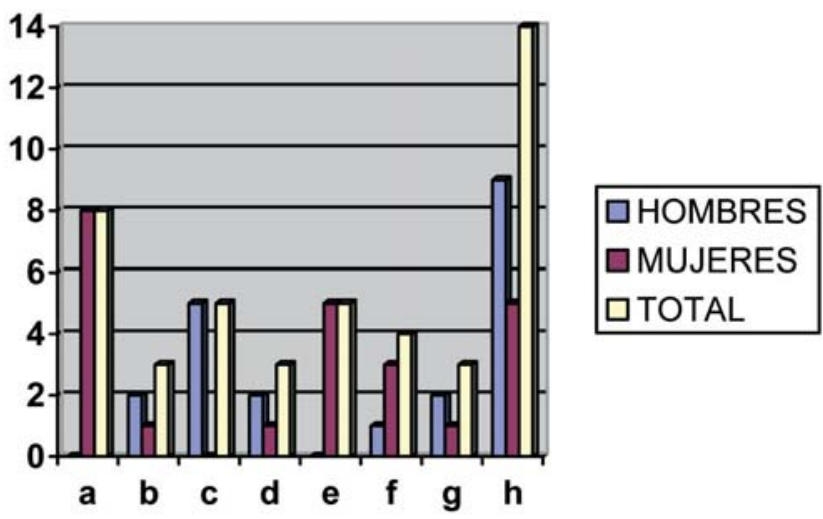

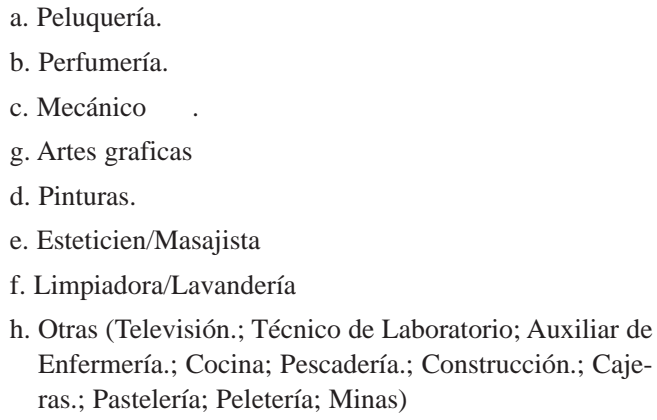
Enfermería.; Cocina; Pescadería.; Construcción.; Cajeras.; Pastelería; Peletería; Minas) 
Figura 3: Distribución de casos por sexo y edad.

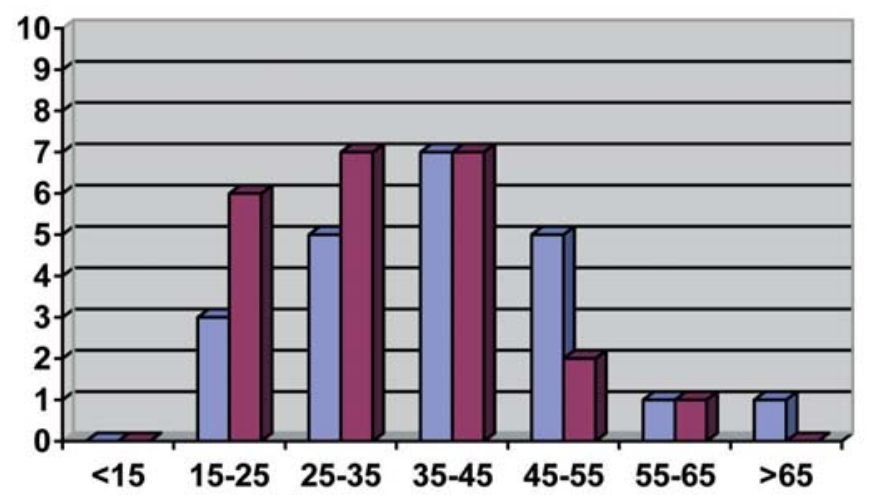

\section{पHombre \\ aMujer}

Figura 4: Principales lesiones por las que acuden a consulta

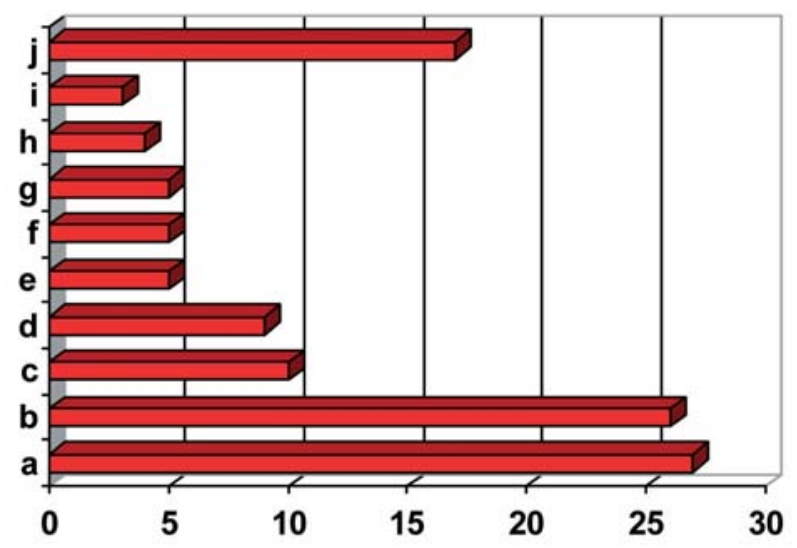

LESIONES:

a) Lesiones Eritematosas.

f) Hiperpigmentación.

b) Descamación.

g) Hiperqueratosis.

c) Eczema.

h) Grietas.

d) Prurito.

i) Vesículas.

e) Pápulas.

j) Otras: liq., Infilt, Xerosis, E. folicular, Habones, L. residual, Onicolisis, Comedones, Queratosis pilar, Máculas, Pústulas, Sequedad.

Figura 5: Diagnósticos clínicos finales.

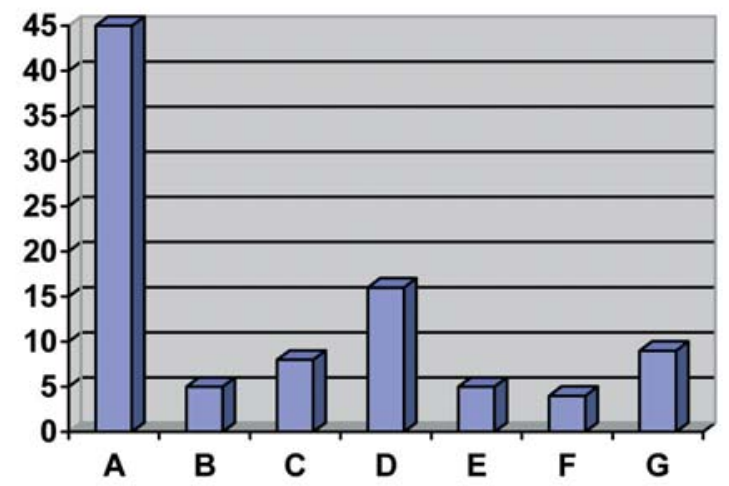
A. Sensibilidad al Kathon.
B. Eczema.
C. Eczema dishidrótico.
D. Eczema alérgico de contacto profesional
E. Dermatitis de contacto irritativa.
F. Dermatitis Atópica.
G. Otras: No lesiones actuales/inespecíficas, Urticaria alérgica de contacto, Psoriasis, D. Seborreica, Enf. Parasitaria




\section{DISCUSION}

Los biocidas son aditivos indispensables en la fabricación de productos con base acuosa, ya que evitan el sobrecrecimiento de microorganismos por ello son utilizados en la manufacturación de cosméticos, productos de limpieza y determinados productos industriales. La metilcloroisotiazolinona (MCI) y la metilisotiazolinona (MI) al igual que otros conservantes del grupo formaldehído o mercuriales, pueden ser sensibilizantes potentes como se ha demostrado por estudios experimentales; el MCI se comporta como más potente sensibilizador que el MI. ${ }^{10,16,17,18}$

Actualmente, tanto la normativa europea para todos los países miembros de la Unión Europea, como la comisión encargada de ingredientes cosméticos americana y la misma casa fabricante, establecen que la concentración debe ser de 15 ppm para los cosméticos rinse-off, y de 7,5 ppm para los stay-on. ${ }^{4}$ La razón por la que siguen manteniéndose tasas de incidencia elevadas en la sensibilización a este alergeno, aceptando de entrada que los fabricantes cumplen la normativa, podemos explicarla porque los mayores consumidores de cosméticos son los pacientes con dermatitis previas quienes al no contar con una barrera cutánea indemne tienen más posibilidades de que el alergeno penetre originando sensibilizaciones, como queda de manifiesto en las revisiones efectuadas en consultas dermatológicas pediátricas, donde el Kathon es el alérgeno más frecuente en este grupo de edad. ${ }^{19,20}$

Otros factores teóricamente influyen en la aparición de esta sensibilización:

1. Es frecuente que los pacientes se apliquen más de un cosmético (jabón y crema) sobre la misma zona y más de una vez al día, por lo que se produce una sumación de la concentración del Kathon.

2. La aplicación puede ser en piel, mucosas o semimucosa tanto sana como con alteraciones de la barrera.

3. La aplicación del tópico bajo oclusión favorece la penetración del alergeno como ocurre con las cremas que se colocan en nalgas y posteriormente se utilizan pañales impermeabilizados. ${ }^{19,20}$

En el año 1992 había registrados 550 nombres comerciales de preparados que contenían MCI/MI en el mercado internacional. La imposibilidad de conocerlos es manifiesta, pero debemos insistir a nuestros pacientes, que lo que deben recordar es el nombre de "Isotiazolina" que es como debe aparecer en el etiquetado desde enero de 1998 en todos los cosméticos, sean de perfumería o farmacia. ${ }^{21,22}$

Además de provocar cuadros de eczema en el lugar de contacto, las isotiazolinas también pueden ocasionar dermatitis de contacto aerotransporta$\mathrm{do}^{23}$, urticarias de contacto, quemaduras con sensibilización posterior y reacciones generalizadas tras exposición a elementos que lo hayan contenido aunque sea después de un largo período, así mismo referir que actualmente está en estudio la posible capacidad teratógena y oncogénica de estas sustancias. $^{24,25}$

Según otros estudios, el Kathon tiene una prevalencia de 4,04\% entre nosotros. Nuestra cifra supera a la de Australia (3,79\%), e Italia (3,1\%), pero esta comprendida dentro del margen o intervalo de las cifras observadas en estudios realizados en otros países. Donde los valores inferiores están representados por el $1,3 \%$ en Portugal y el $0,2 \%$ en Turquía. ${ }^{2,17}$ La reducción del porcentaje utilizado en cosméticos en muchos países ha hecho que disminuyera su prevalencia.

Estudios confirman una elevada sensibilidad en nuestro país, lo que indicaría que podrían ser los productos locales los responsables de esta incidencia mayor que en otros países

En el año 1990 en este mismo Servicio de Dermatología Laboral realizó el primer estudio de sensibilización al Kathon CG en nuestro país, encontrando los primeros 22 casos que suponían el 3,5\% de las positividades. En otros estudios realizados, la cifra de sensibilización de los años comprendidos entre 1990-1997 es del 2,89\% con oscilaciones que varían entre el 1 y el $5 \%{ }^{26}$

En cuanto a la relación del Kathon como sustancia sensibilizante en el mundo laboral, debemos estudiar una serie de puntos, que podrán ser objeto de estudios posteriores:

- El Kathon está presente en numerosas sustancias que se usan en el mundo laboral.

- En este estudio, podemos ver una relación entre trabajo y Kathon presente en el $41.5 \%$ de las sensibilizaciones. No debemos perder de vista que además de este $41,5 \%$ queda un $15,11 \%$ de sensibilizaciones en las cuales no esta claro si realmente la sensibilización se debe al trabajo, pero lo que sí está claro, es que el trabajo influye bien como sensibilizante ini- 
cial o como promotor del cuadro dermatológico.

- Las profesiones más afectadas (peluquería, perfumería, mecánicos, Esteticien/cosmética y limpieza), a su vez son las que más utilizan productos que contienen Kathon como conservante (jabones, champús, detergentes, cremas etc. $)^{5,7,9,10,12,14}$

Por otro lado se puede observar un ligero aumento en el número de hombres afectados, lo que indica que la fuente es más laboral que privada, ya que los pacientes que vemos son en su mayoría remitidos por problemas profesionales.

\section{BIBLIOGRAFIA}

1. Hunziker N. The "Isothiazolinone story" Dermatology, 1992; 184(2):85-6.

2. De Groot A.C. Methylisothiazolinone/Methylchoroisotiazolinone (kathon CG) Allergy: An Updated review. Am J Contac Dermatitis 1990; 3:151-156.

3. O'Driscoll JB, Beck MH. Occupational allergic contac dermatitis from Kathon WT. Contact Dermatitis. 1988 jul; 19(1):63.

4. Rastogi SC. Kathon CG and cosmetic products. Contac Dermatiti. 1990 Mar;22(3):155-60.

5. Fischer T, Bohlin S, Edling C, Rystedt I, Wieslander G. Skin disease and contact sensitivity in house painters using water-based, glues and putties. Contact Dermatitis 1995; 32: 39-45.

6. Rycroft R J G, Neil V S. Allergic contact dermatitis from MCI/MI biocide in a printer. Contact Dermatitis 1992; 26: 142-143.

7. Bruynzeel D P, Verburgh C A. Occupational dermatitis from isothiazolinones in disel oil. Contact Dermatitis 1996; 34: 64-65.

8. Pazzaglia M, Vincenzi C, Gasparri F, Tosti A. Occupational hypersensitivity to isothiazolinone derivatives in a radiology technician. Contact Dermatitis 1996; 34: 143.

9. Guimaraens D, Conde-Salazar L, González M A.Allergic contact dermatitis on the hands from chloromethylisothiazolinone in moist toilet paper.Contact Dermatitis 1996; 35: 254.

\section{CONCLUSIONES}

Según nuestro estudio, la sensibilización al Kathon en los tres últimos años ha estado entorno al $3,68 \%$, cifra que concuerda con estudios anteriores.

En este estudio, podemos ver una relación entre trabajo y Kathon presente en el $41.5 \%$ de las sensibilizaciones. No debemos perder de vista que además de este $41,5 \%$ queda un $15,11 \%$ de sensibilizaciones en las cuales no esta claro si realmente la sensibilización se debe al trabajo, pero lo que sí está claro es que influye bien como sensibilizante inicial o como promotor del cuadro dermatológico. ${ }^{2,17,26}$
10. Gebhardt M, Looks A, Hipler U C. Urticaria caused by Type IV sensitization to isothiazolinones. Contact Dermatitis 1997; 36: 314.

11. Primka E J, Taylor J S. Three cases of contact allergy after chemical burns from methylclhloroisothiazolinone/methylisothiazolinone: one with concomitant allergy to methyldibromoglutaronitrile/phenoxyethanol. Am J Contact Dermatitis 1997;8: 43-46.

12. Herzong J, Dunne J, Aber R, Claver M, Marks JG Jr. Milk tester's dermatitis. J Am Acad Dermatol. 1988 Sep; 19(3):503-8

13. Moss JN. Occupacional dermatitis in milk industry worker due to Kathon CG. Dermatitis, 2004 Dec;15(4)215; author replay 215.

14. Guo YL, Wang BJ, Lee JY, Chou SY. Occupational hand dermatoses of hairdressers in Tainan City. Occup Environ Med. 1994 kOct; 51(10): 689-92

15. De Groot A C, Baar T J M, Terpstra H, WeylandW. Contact allergy to moist toilet paper. Contact Dermatitis 1991; 24: 135.

16. Fernández De Corres L, Navarro J A, Gastaminza G, Del Pozo M D. An unusual case of sensitization tomethylchloro- and methyl-isothiazolinone (MCI/MI). Contact Dermatitis 1995; 33: 215.

17. Menné T, Frosch P J, Veien N K, Hannuksela M, Björkner B, Lachapelle J M, et al. Contact sensitization to 5-chloro-2methyl-4-isothiazolin-3-oneand 2-methyl-4isothiazolin-3 one and 2-methyl-4-isothiazolin-3-one (MCI/MI). A European multicentrestudy. Contact Dermatitis 1991; 24: 333-341. 
18. Marks J G, Moss J N, Parno J F, Adams R M, Belsito D V, De Leo F, et al.: Methylchlorothiazolinone/ Methylisothiazolinone (Kathon CG) Biocide: Second. United States Multicenter Study of HumanSkin Sensitization. Am J Contact Dermatitis 1990; 4:87-89.

19. De Groot A C. Vesicular dermatitis of the hands secondary to perianal allergic contact dermatitis caused by preservatives in moistened toilet tissues. Contact Dermatitis 1997; 36: 173.

20. Conti A, Motolese A, Manzini B M, Seidenari S. Contact sensitization to preservatives in children. Contact Dermatitis 1997; 37: 35-52.

21. Gruvberger B, Persson K, Bjorkner B, Bruze M, Dahlquist I, Fregert S. Demonstration of Kathon CG in some comercial products. Contac Dermatitis. 1986 Jul;15(1):24-7.
22. Nielsen H. Occupational exposure to isothiazolinones. A study based on a product register. Contact Dermatitis 1994; 31: 18-21.

23. Schubert H. Airborne contact dermatitis due to methylchloro-and methylisothiazolinone (MCI/MI). Contact Dermatitis 1997; 36: 274.

24. Longacre S L. Genetic toxicity and carcinogenicity studies of Kathon. Environ Mol Mutagen 1997; 29, 3: 331-333.

25. Comnor TH, Tee PG, Afshar M, Comnor KM. Mutagenicity of cosmetic products containing Kathon Mutagen 1997;29(3):330

26. Hasson A, Guimaraens D, Conde-Salazar L. Patch test sensitivity to the preservative Kathon CG in Spain Contact Dermatitis 1990; 22: 257-261. 
